13,01

\title{
Квантово-механический подход к определению энергии активации поверхностной диффузии
}

\author{
(С Э.Ф. Штапенко, В.В. Титаренко , В.А. Заблудовский, Е.О. Воронков
}

Днепровский национальный университет железнодорожного транспорта им. акад. В. Лазаряна,

Днепр, Украина

『 E-mail: tytarenko.valentina@gmail.com

Поступила в Редакцию 18 июня 2020 г.

В окончательной редакции 18 июня 2020 г.

Принята к публикации 22 июня 2020 г.

\begin{abstract}
Предложен квантово-механический подход к определению энергии активации поверхностной диффузии для адсорбированных атомов меди, никеля, цинка и железа на медной подложке при электрокристаллизации для различных перенапряжений подложки. Расчет энергии активации поверхностной диффузии выполнен через полную энергию кристалла. Увеличение энергии активации поверхностной диффузии при увеличении потенциала поверхности связано с увеличением энергии связи ад-атома с подложкой.
\end{abstract}

Ключевые слова: энергия активации поверхностной диффузии, ад-атом, электрокристаллизация, перенапряжение.

DOI: 10.21883/FTT.2020.11.50074.129

\section{1. Введение}

Одним из важных этапов во многих поверхностных процессах, особенно при электрокристаллизации [1], является поверхностная диффузия, которая заключается в массопереносе по поверхности твердого тела.

Поверхность электрода, по которой происходит поверхностная диффузия при электрокристаллизации, является эквипотенциальной поверхностью, и движущей силой такого процесса является не только тепловое движение, а и избыточная энергия ад-атома.

Диффузия по поверхности металлов в значительной степени зависит от структуры поверхности [2]. Реальный кристалл имеет неоднородную поверхность и характеризуется сложным микрорельефом. В реальных условиях поверхность содержит множество чужеродных атомов, которые адсорбировались или вышли на поверхность из твердой фазы.

Энергия активации $\left(E_{S}\right)$ является важнейшим параметром поверхностной диффузии. Существуют различные методы экспериментального исследования поверхностной диффузии, которые фиксируют результат перемещения частиц (меченые атомы, определение работы выхода).

Для того, что бы определить энергию активации необходимо знать поверхностный энергетический рельеф системы ,подложка-ад-атом“. Поэтому расчет $E_{S}$ является чрезвычайно сложной задачей. Наиболее популярным, в настоящее время, теоретическим методом расчета $E_{S}$ для металлов является метод внедренного атома (embedded atom method, или EAM) [3] и когезионного приближения. В работе [4] предложен подход к вычислению энергии активации поверхностной диффузии, который основан на когезионном приближении к расчету адсорбционных свойств атомов. Получена удо- влетворительная корреляция между результатами расчета $E_{S}$ для диффузии атомов всех переходных, а также редкоземельных металлов по поверхности кристаллической плоскости (hkl) (110) вольфрама и имеющимися немногочисленными экспериментальными данными.

В настоящей работе, на основе развития когезионного приближения, приводятся результаты теоретических расчетов энергии активации поверхностной диффузии для адсорбированных атомов меди, никеля, цинка и железа на медной подложке при электрокристаллизации.

\section{2. Модели и методы расчета}

С целью рассмотрения движения ад-атома на кристаллической поверхности подложки, представим поверхность в виде периодического массива адсорбционных положений, которые соответствуют положениям минимумов энергии. Поверхностная диффузия может быть рассмотрена как перескок из одной поверхностной ячейки в соседнюю $[5,6]$. Будем предполагать, что в процессе перескока из одной поверхностной ячейки в другую (соседнюю) длина адсорбционной связи остается неизменной.

Поверхностная диффузия при электрокристаллизации рассматривается как перемещение ад-атома осаждаемого металла по поверхности подложки, при котором положение ад-атома изменяется от одного узла кристаллической решетки до другого через равные расстояния.

Рассмотрим поверхностную диффузию по подложке ад-атомов никеля и меди, которые имеют ГЦК-решетку. При начальных стадиях кристаллизации и в процессе роста пленок подложка не имеет ступеней роста.

Поверхность ГЦК-кристалла (плоскость (100)) приведена на рис. 1. Ад-атом расположен в узле кристалличе- 


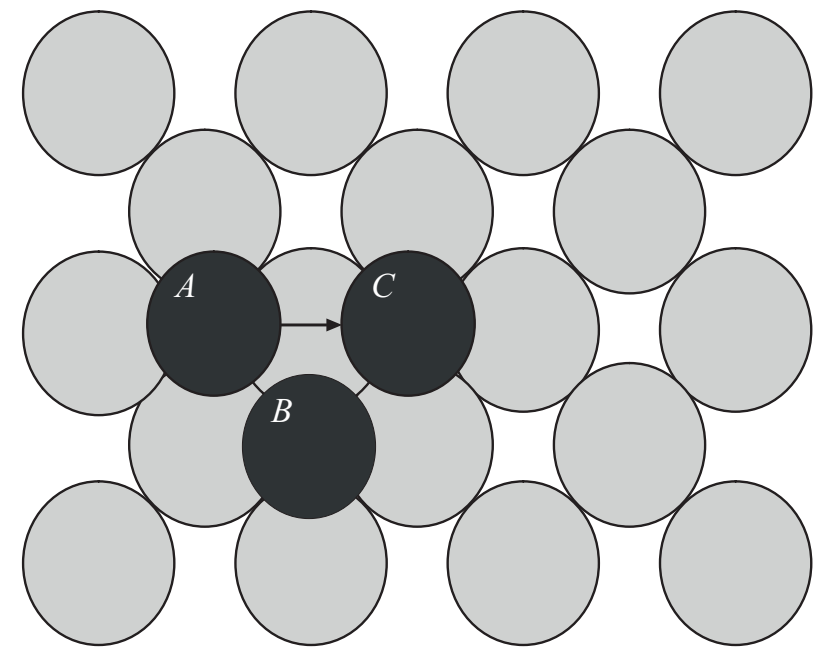

Рис. 1. Диффузия по поверхности ГЦК-кристалла, плоскость (100): $A-$ начальное положение ад-атома, $B$ и $C-$ конечные положения ад-атома.

ской решетки $A$ в плоскости (200) на расстоянии $0.5 a$ ( $a$ - параметр кристаллической решетки).

Возможна диффузия ад-атома в ближайшее положение $B$. Длина перескока между узлами $A$ и $B$ равна $b=a \sqrt{0.5}$. Также рассматривается перемещение между узлами $A Q$ и $C$ с длиной перескока $b=a$.

В отличие от когезионного приближения, с целью определения энергии активации был проведен расчет полной энергии подложки с ад-атомом в каждом из этих положений. Для этого проведено построение геометрической модели кристалла с ад-атомом, в которой выбор числа атомов в кристаллической решетке основывался на необходимости взаимодействия ад-атома с атомами подложки, как минимум, в пяти координационных сфеpax. Следовательно, энергия активации поверхностной диффузии будет определяться как разность полной энергии кристалла с ад-атомом в равновесном адсорбционном состоянии, в котором энергия минимальна $\left(W_{\min }\right)$, и в переходной седловой точке, в котором энергия максимальна $\left(W_{\max }\right)[7]$ :

$$
E_{S}=W_{\max }-W_{\min } .
$$

Расчет полной энергии кристалла с адсорбированными атомами проводился с помощью одного из наиболее распространенных методов расчета электронной структуры атомов, молекул, кластеров, твердых тел и т.п. теории функционала плотности (ТФП) $[8,9]$. ТФП сочетает в себе достаточно высокую точность получаемых результатов, конкурирующей, в ряде случаев, с точностью ab initio методов учета электронной корреляции, и с достаточно умеренными требованиями к вычислительным ресурсам, которые позволяют проводить расчеты систем, состоящих из сотен атомов и представляющих интерес для современной нанотехнологии [10].

Кроме того, метод ТФП используется для исследования адсорбционных характеристик переходных метал- лов [11-13], кинетика электрокристаллизации которых исследуется в данной работе.

Согласно теории функционала плотности полная энергия $(W)$ определяется выражением

$$
\begin{aligned}
W= & U-\frac{1}{2} \sum_{i=1}^{N_{\text {occup }}} \int \phi_{i}^{*}(r) \nabla^{2} \phi_{i}(r) d r-\sum_{K} \int \frac{\rho(r)}{\left|r-R_{K}\right|} d r \\
& +\frac{1}{2} \iint \frac{\rho(r) \rho\left(r^{\prime}\right)}{\left|r-r^{\prime}\right|} d r d r^{\prime}+E_{X C}[\rho],
\end{aligned}
$$

где $Z_{K}$ и $R_{K}$ - заряд и пространственные координаты неподвижного ядра с номером $K$ соответственно и электронная плотность

$$
\rho(r)=\sum_{i=1}^{N_{\text {occup }}}\left|\varphi_{i}(r)\right|^{2},
$$

где $\varphi_{i}(r)$ - одноэлектронная Кон-Шэмовская (молекулярная) орбиталь.

Выражение (2) в свернутом виде имеет вид

$$
W=U+T_{S}\left\{\phi_{i}(r)\right\}_{i=1}^{N_{\text {occup }}}+V_{n e}[\rho]+J[\rho]+E_{X C}[\rho],
$$

где $U-$ потенциальная энергия взаимодействия ядер, $T_{S}$ - электронная кинетическая энергия, $V_{n e}-$ притяжение электронов к ядрам, $J-$ классический вклад в энергию межэлектронного отталкивания и $E_{x c}-$ обменно-корреляционный функционал, включающий статическую электронную корреляцию.

Многочисленные исследования характеристик молекул и кластеров методом ТФП [14-16] показали хорошие результаты при правильном выборе обменно-корреляционного функционала. В работах $[17,18]$ показано, что наиболее подходящим для расчетов структурных и термохимических характеристик комплексов металлов является трехпараметрический гибридный функционал B3LYP [19-21]. Известно также, что применение гибридных обменно-корреляционных функционалов в теории функционала плотности позволяет успешно рассчитывать структурные и электронные характеристики комплексов переходных и тяжелых металлов с приемлемыми затратами машинного времени [22-25].

Существует ряд обзорных и оригинальных работ, которые посвящены вопросу определения типа функционала, позволяющего наиболее точно рассчитать характеристики переходных металлов [26-33]. Показано, что при использовании функционала BЗLYP для расчета характеристик структуры переходных металлов, точность обеспечивается на уровне $a b$ initio методов.

Осуществленный нами выбор гибридного функционал B3LYP обоснован проведенными тестированиями обменно-корреляционного функционала М062Х и гибридных функционалов B3LYP и PBE1PBE [7].

Кроме того, необходимо учитывать, что в поставленных в работе задачах, таких как расчет энергий связи, определение энергий активации поверхностной диффузии, используются не абсолютные значения энергий, а их разностные величины. 


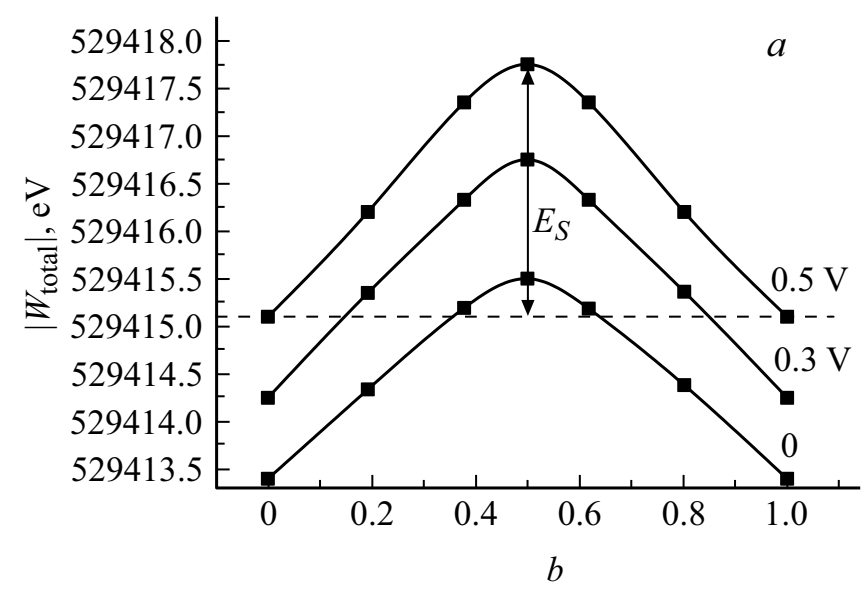

Рис. 2. Зависимости значений полной энергии кристалла диффундирующего ад-атома при диффузии из положения $A$ в $B$

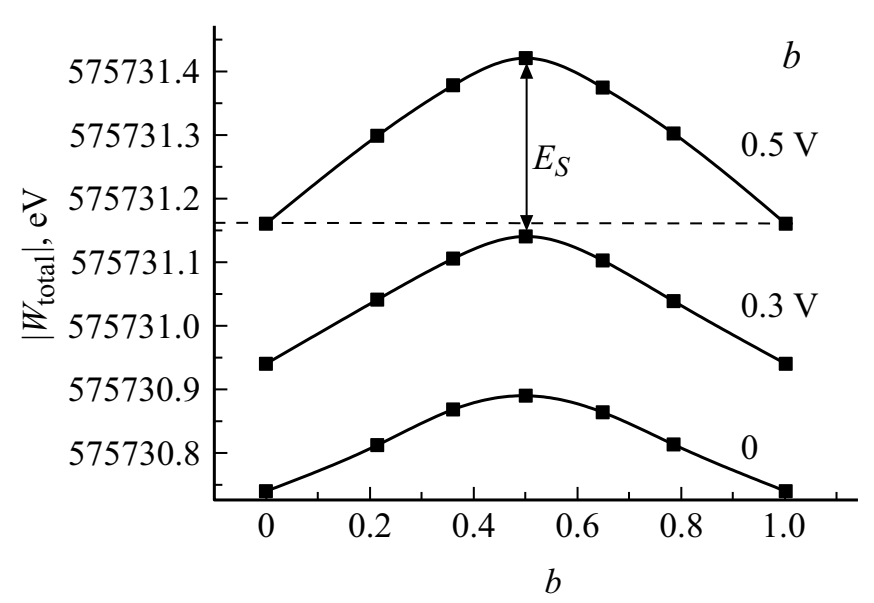

$(W)$ меди от длины перескока $(b)$ для различных положений $(a)$ и $A$ в $C(b)$.
Энергетические величины были рассчитаны для металлов, для которых важную роль играет взаимодействие валентных электронов. По данному принципу осуществлялся выбор базисного набора. Для описания таких взаимодействий используются валентно-расщепленные наборы базисных орбиталей. Наиболее рекомендуемым для описания взаимодействия в многоэлектронных системах является базис 6-31g или расширенный 6-31-g $(d)$, который содержит атомные орбитали $d$-типа для учета поляризации электронной плотности тяжелых металлов.

Для металлов с еще большим количеством электронов рекомендуется валентно-расщепленный базисный набор lanl2dz с эффективным потенциалом остова (Effective Core Potentials - ECP) [34].

Для проведения расчетов энергий в конденсированном состоянии [35-37] был использован пакет программ GAUSSIAN 03 [38]. При выполнении расчетов температура выбиралась $95 \mathrm{~K}$, давление $10^{5} \mathrm{~Pa}$.

\section{3. Результаты и обсуждение}

Рассмотрим процесс электрокристаллизации при потенциостатическом режиме, при котором на подложке поддерживается постоянное перенапряжение $(\eta)$, а именно - самодиффузию ад-атомов меди на медной подложке.
Результаты расчетов значений полной энергии кристалла $(W)$ меди по формуле (4), для различных положений диффундирующего ад-атома никеля при диффузии из положения $A$ в $B$, приведены на рис. 2 .

В табл. 1 приведены значения энергии активации поверхностной диффузии для адсорбированных атомов никеля и цинка, рассчитанные по формуле (1), для различных перенапряжений подложки.

Результаты расчетов, приведенные в табл. 1, показывают, что, во-первых, значение энергии активации поверхностной диффузии зависит от потенциала поверхности (подложки в случае электрокристаллизации): с увеличением потенциала значение энергии активации также растет. Так, например, для меди при увеличении перенапряжения от $0.1 \mathrm{~V}$ до $0.2 \mathrm{~V}$ величина $E_{S}$ возрастает от $0.19 \mathrm{eV}$ до $0.26 \mathrm{eV}$ для перескока $A B$ и от $1.76 \mathrm{eV}$ до $2.11 \mathrm{eV}$ для перескока $A C$. Во-вторых, значение энергии активации зависит от длины перескока. С увеличением длины перескока, при одинаковом количестве ближайших соседей, энергия активации также увеличивается. Так для перескоков $A B$ и $A C\left(b_{A C}=\sqrt{2} b_{A B}\right)$ значения $E_{S}$ : для меди при потенциалах $0.2 \mathrm{~V}, 0.1 \mathrm{~V}$ и $0-0.26 \mathrm{eV}$ и $2.107 \mathrm{eV}, 0.19 \mathrm{eV}$ и $1.76 \mathrm{eV}, 0.16 \mathrm{eV}$ и $1.13 \mathrm{eV}$.

Увеличение энергии активации поверхностной диффузии при увеличении потенциала поверхности, в первую очередь, связано с увеличением энергии связи ад-атома с подложкой [39-41].

Таблица 1. Значения энергии активации поверхностной диффузии для различных перенапряжений на подложке и разных направлений перескока

\begin{tabular}{c|c|c|c|c|c|c|c|c|c|c|c}
\hline \multicolumn{2}{c|}{ Величина } & \multicolumn{3}{c|}{$\mathrm{Cu}$} & \multicolumn{3}{c|}{$\mathrm{Ni}$} & \multicolumn{2}{c}{$\mathrm{Zn}$} \\
\hline \multicolumn{2}{c|}{$\eta, \mathrm{V}$} & 0.2 & 0.1 & 0 & 0.5 & 0 & 0.4 & 0 & 0.3 & 0 \\
\hline \multicolumn{2}{c|}{$E_{S}}$, & $A B$ & 0.26 & 0.19 & 0.16 & 0.53 & 0.24 & 0.29 & 0.15 & 0.35 & 0.18 \\
$\mathrm{eV}$ & $A C$ & 2.11 & 1.76 & 1.13 & 2.3 & 1.9 & 2.3 & 1.3 & 2.2 & 1.3
\end{tabular}




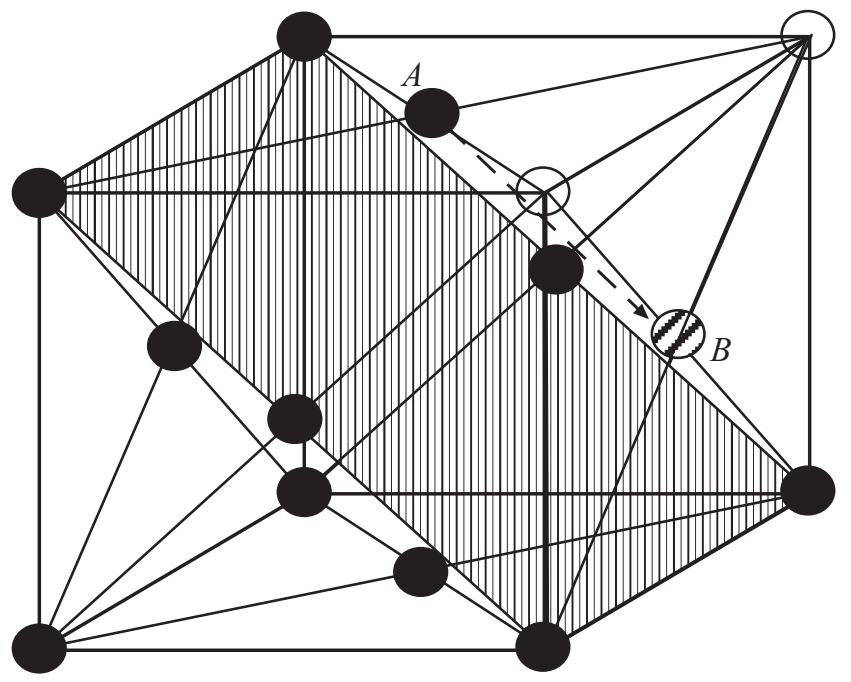

Рис. 3. Схема диффузии по поверхности ГЦК-кристалла, плоскость (110): $A$ - начальное положение ад-атома, $B-$ конечное положение ад-атома.

Результаты расчетов приведены для диффузии по плоскости (100) для ГЦК-решетки. Установлена хорошая корреляция между полученными значениями энергий активации и результатами других исследователей $[3,5,42]$.

Результаты расчетов значений $E_{S}$ самодиффузии ад-атомов меди $(\eta=0.2 \mathrm{~V})$, никеля $(\eta=0.5 \mathrm{~V})$, цинка $(\eta=0.4 \mathrm{~V})$ и железа $(\eta=0.3 \mathrm{~V})$ на медной подложке по плоскости (110) (рис. 3) приведены в табл. 2.

Рассмотрим случай, когда диффузия происходит около ступени роста. Атомы, показанные на рис. 4 серым цветом, образуют ступень роста и расположены в плоскости (200). Самодиффузия ад-атома происходит вдоль ступени роста.

В таком случае, при такой диффузии, практически отсутствует зависимость между энергией активации и потенциалом подложки. Так, например, при уменьшении потенциала от $0.2 \mathrm{~V}$ до нуля, энергия активации меняется от $1.56 \mathrm{eV}$ до $1.5 \mathrm{eV}$. Увеличение энергии активации связано, в первую очередь, с увеличением числа ближайших соседей, что также сказывается на увеличении энергии связи.

Результаты расчетов показывают, что самодиффузия ад-атомов около неровностей кристаллических поверх-

Таблица 2. Значения энергии активации поверхностной диффузии ад-атомом меди $(\eta=0.2 \mathrm{~V})$, никеля $(\eta=0.5 \mathrm{~V})$, цинка $(\eta=0.4 \mathrm{~V})$ и железа $(\eta=0.3 \mathrm{~V})$ на медной подложке по плоскости (110)

\begin{tabular}{c|c|c|c|c}
\hline Величина & $\mathrm{Cu}$ & $\mathrm{Ni}$ & $\mathrm{Zn}$ & $\mathrm{Fe}$ \\
\hline$\eta, \mathrm{V}$ & 0.2 & 0.5 & 0.4 & 0.3 \\
$E_{S}, \mathrm{eV}$ & 0.39 & 0.77 & 0.42 & 0.55
\end{tabular}

ностей затруднена по сравнению с гладкой поверхностью. Следовательно, при электрокристаллизации на гладкой поверхности ад-атом имеет возможность диффундировать по поверхности, тогда как, около ступени роста вероятность его диффузии заметно уменьшается.

Рассмотрим диффузию, схема которой приведена на рис. 5. В данном случае ад-атом расположен над атомной неоднородностью в виде ступени роста и диффундирует по поверхности, занимая более устойчивое положение в плоскости (200), тем самым продолжая рост.

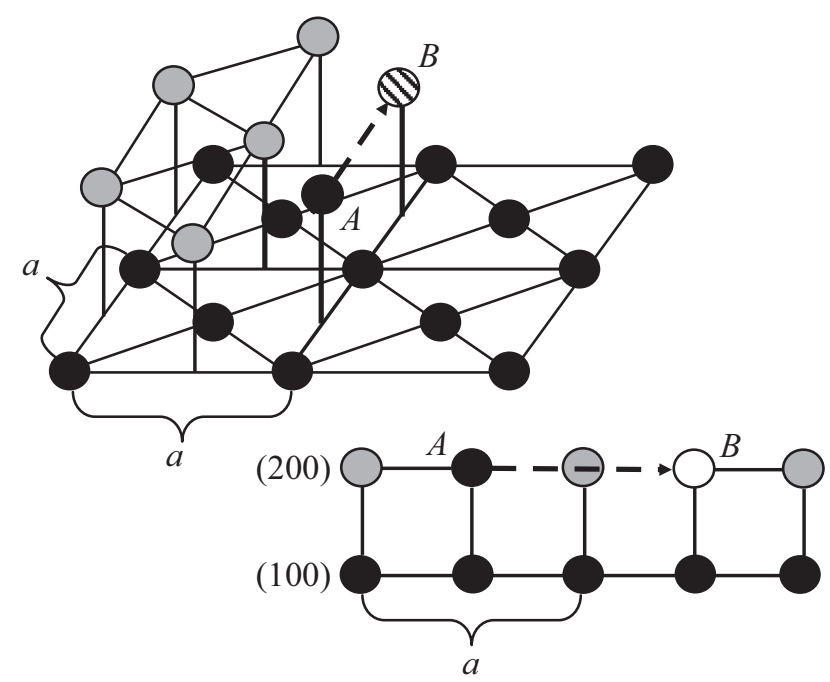

Рис. 4. Схема диффузии по поверхности ГЦК-кристалла, плоскость (100) и ступень роста (200): $A$ - начальное положение ад-атома, $B-$ конечное положение ад-атома.

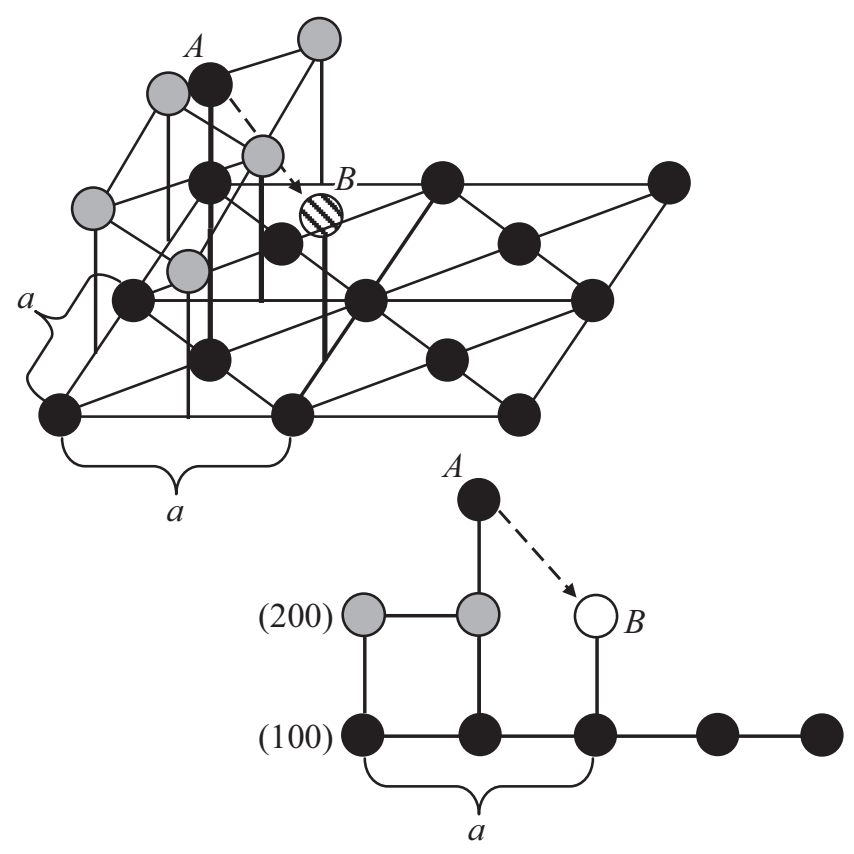

Рис. 5. Схема диффузии по поверхности ГЦК-кристалла, диффундирующий ад-атом над ступенью роста (200): $A$ начальное положение ад-атома, $B-$ конечное положение ад-атома. 


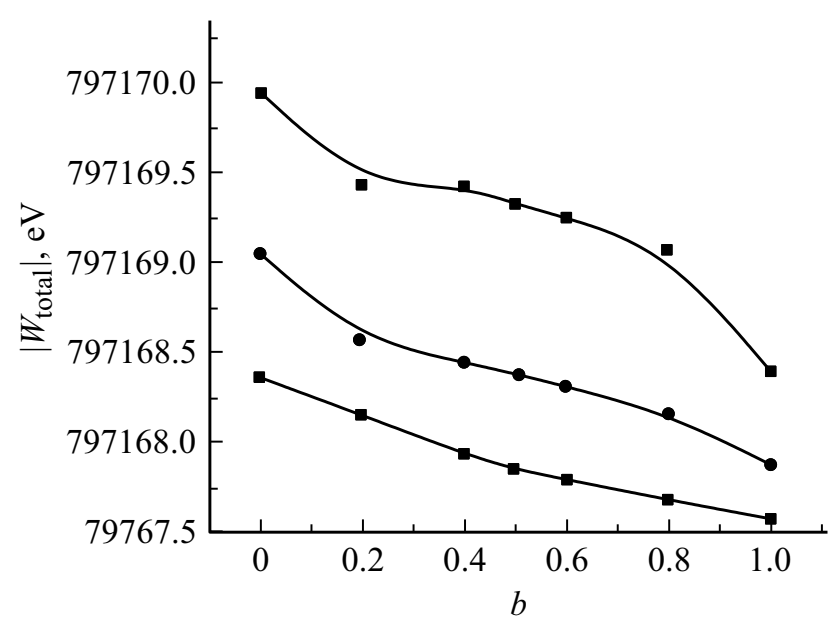

Рис. 6. Зависимость полной энергии кристалла $\left(W_{\text {total }}\right)$ меди для различных положений диффундирующего ад-атома меди при диффузии из положения $A$ (диффундирующий ад-атом над ступенью роста) в $B$.

Результаты расчетов полной энергии кристалла $\left(W_{\text {total }}\right)$ меди для различных положений диффундирующего ад-атома меди при диффузии из положения $A$ (диффундирующий ад-атом над ступенью роста) в $B$ приведены на рис. 6.

Из данных рис. 6 следует, что значения энергии кристалла в начальном $(A)$ и конечном $(B)$ положениях при такой диффузии не равны, в отличие от всех предыдущих случаев, в которых ад-атом после диффузии по поверхности занимал равнозначное новое положение. В данном рассматриваемом случае ад-атом стремится занять положение, соответствующее наименьшей энергии кристалла в целом и наибольшей энергии связи самого ад-атома. В случае превышения энергия связи ад-атома над значением его энергии связи в положении $A$ такая диффузия возможна и происходит без преодоления потенциального барьера. Однако, в таком случае полученные значения энергии кристалла с ад-атомом в начальном и конечном положениях можно рассматривать как значения энергии активации поверхностной диффузии из положения $B$ в положение $A$.

\section{4. Выводы}

Предложенный квантово-механический подход позволяет рассчитать значения энергии активации поверхностной диффузии для различных абсорбированных атомов. Особенностью данного метода является учет потенциала поверхности, что совершенно невозможно учесть в других теоретических методах, в частности, в когезионном приближении. Последнее обстоятельство особенно важно при электрокристаллизации на подложке. Показано, что полученные значения $E_{S}$ сильно зависят от потенциала и от геометрии поверхности.
Полученные данные удовлетворительно коррелируют с результатами других исследователей.

\section{Список литературы}

[1] К. Оура, В.Г. Лифшиц, А.А. Саранин, А.В. Зотов, М. Катаяма. Введение в физику поверхности. Наука, М. (2006). $490 \mathrm{c}$.

[2] Я.Е. Гегузин. Поверхностная диффузия и растекание. Наука, М. (1969). С. 11-77.

[3] C.M. Chans, C.M. Wei, S.P. Chen. Phys. Rev. B 54, 17083 (1996).

[4] S.Yu. Davydov, S.K. Tikhonov. Surf. Sci. 275, 137 (1992).

[5] С.Ю. Давыдов. ФТТ 41, 11 (1999).

[6] С.Ю. Давыдов. Письма в ЖТФ 24, 70 (1998).

[7] Э.Ф. Штапенко. В кн. Тез. докл. 75 Междунар. науч.-практ. Конф. „Проблемы и перспективы развития железнодорожного транспорта“ Днепропетровск (2015). C. 415.

[8] R.G. Parr, W. Yang. Density-Functional Theory of Atoms and Molecules. Oxford University Press, N. Y. (1989). 333 p.

[9] W. Koch, M.C. Holthausen. Chemists Guide to Density Functional Theory. 2nd Ed. Wiley-VCH, N. Y. (2001). 293 p.

[10] А.В. Арбузников. Журн. структур. химии 48, 38 (2007).

[11] M.K. Sabbe, M.F. Reyniersa, K. Reuter. Catal. Sci. Technol. 2, 2010 (2012).

[12] N. Lopez, N. Almora-Barrios, G. Carchini, P. Błoński, L. Bellarosa, R. Garcìa-Muelas, G. Novell-Leruth, M. GarcìaMota. Catal. Sci. Technol. 2, 2405 (2012).

[13] T.C. Allison, Y.Y.J. Tong. Phys. Chem. Chem. Phys. 13, 12858 (2011).

[14] Н.Ф. Степанов. Квантовая механика и квантовая химия. Мир, М. (2001). 519 с.

[15] K. Burke. J. Chem. Phys. 136, 150901 (2012).

[16] A.D. Becke. J. Chem. Phys. 140, 18A301 (2014).

[17] G. Schreckenbach, P.J. Hay, R.L. Martin. Inorg. Chem. 37, 4442 (1998).

[18] G. Schreckenbach, P.J. Hay, R.L. Martin J. Comput. Chem. 20, 70 (1999).

[19] A.D. Becke. J. Chem. Phys. 98, 5648 (1993).

[20] C. Lee, W. Yang, R.G. Parr. Phys. Rev. B. 37, 785 (1988).

[21] B. Miehlich, A. Savin, H. Stoll, H. Preuss. Chem. Phys. Lett. 157, 200 (1989).

[22] D.A. Keire, Y.H. Jang, L. Li, S. Dasgupta, W.A. Goddard, J.E. Shively. Inorg. Chem. 40, 4310 (2001).

[23] W. Kohn, A.D. Becke, R.G. Parr. J. Phys. Chem. 100, 12974 (1996).

[24] J. Andzelm, J. Labanowski. Density Functional Methods in Chemistry. Heidelberg, Springer -Verlag (1991). 443 p.

[25] В.Ю. Бузько, И.В. Сухно, А.А. Полушин, В.Т. Панюшкин. Журн. структур. химии 47, 249 (2006).

[26] M.C. Holthausen. J. Comput. Chem. 26, 1505 (2005).

[27] F.S Legge, G.L. Nyberg, J.B. Peel. J. Phys. Chem. A. 105, 7905 (2001).

[28] N.E. Schultz, Y. Zhao, D.G. Truhlar. J. Phys. Chem. A. 109, 4388 (2005).

[29] N.J. Mayhall, K. Raghavachari, P.C. Redfern, L.A. Curtiss. J. Phys. Chem. A. 113, 5170 (2009).

[30] J. Handzlik. Chem. Phys. Lett. 469, 140 (2009).

[31] E.A. Amin, D.G. Truhlar. J. Chem. Theory Comput. 4, 75 (2008). 
[32] J. Rogal, K. Reuter, M. Scheffler. Phys. Rev. B 75, 205433 (2007).

[33] S.S. Tafreshi, A. Roldan, N.Y. Dzade, N.H. de Leeuw. Surf. Sci. 622, 1 (2014).

[34] П.В. Серба, С.П. Мирошниченко, Ю.Ф. Блинов. Квантовохимические расчеты в программе ОА11881А1Ч. Изд-во ТТИ ЮФУ, Таганрог (2012). 100 с.

[35] Е.В. Бутырская. Компьютерная химия: Основы теории и работа с программами GAUSSIAN и GAUSSVIEW. Солонпресс. (2011). 224 c.

[36] J. Foresman. Exploring Chemistry with electronic structure methods. Gaussian, Inc. (1996). 302 p.

[37] J.W. Ochterski. Thermochemistry in Gaussian. Gaussian, Inc. (2000). 19 p.

[38] Gaussian 03, Revision C.02, M.J. Frisch, G.W. Trucks, H.B. Schlegel, G.E. Scuseria, M.A. Robb, J.R. Cheeseman, Jr.J.A. Montgomery, T. Vreven, K.N. Kudin, J.C. Burant, J.M. Millam, S.S. Iyengar, J. Tomasi, V. Barone, B. Mennucci, M. Cossi, G. Scalmani, N. Rega, G.A. Petersson, H. Nakatsuji, M. Hada, M. Ehara, K. Toyota, R. Fukuda, J. Hasegawa, M. Ishida, T. Nakajima, Y. Honda, O. Kitao, H. Nakai, M. Klene, X. Li, J.E. Knox, H.P. Hratchian, J.B. Cross, V. Bakken, C. Adamo, J. Jaramillo, R. Gomperts, R.E. Stratmann, O. Yazyev, A.J. Austin, R. Cammi, C. Pomelli, J.W. Ochterski, P.Y. Ayala, K. Morokuma, G.A. Voth, P. Salvador, J.J. Dannenberg, V.G. Zakrzewski, S. Dapprich, A.D. Daniels, M.C. Strain, O. Farkas, D.K. Malick, A.D. Rabuck, K. Raghavachari, J.B. Foresman, J.V. Ortiz, Q. Cui, A.G. Baboul, S. Clifford, J. Cioslowski. B.B. Stefanov, G. Liu, A. Liashenko, P. Piskorz, L. Komaromi, R.L. Martin, D.J. Fox, T. Keith, M.A. Al-Laham, C.Y. Peng, A. Nanayakkara, M. Challacombe, P.M.W. Gill, B. Johnson, W. Chen, M.W. Wong, C. Gonzalez, J.A. Pople. Gaussian, Inc., Wallingford CT. (2004).

[39] Е.П. Штапенко, В.О. Заблудовський, В.В. Титаренко. Фізика і хімія твердого тіла 16, 520 (2015).

[40] Е.П. Штапеко, В.О. Заблудовський, В.В. Дудкіна. Фізика і хімія твердого тіла 4, 618 (2014).

[41] Е.П. Штапенко, В.О. Заблудовський, Е.О. Воронков, В.В. Дудкіна. Фізика і хімія твердого тіла 15, 34 (2014).

[42] A.G. Naumovets. Surf. Sci. Rep. 4, 365 (1985).

Редактор Ю.Э. Китаев 